\title{
AERIAL MAGNETIC SENSING WITH AN UAV HELICOPTER
}

\author{
Christoph Eck* and Benedikt Imbach $^{\dagger}$ \\ Aeroscout GmbH \\ and \\ Lucerne University of Applied Sciences and Arts \\ Technikumstrasse 21 \\ 6048 Horw, Switzerland
}

email: eck@aeroscout.ch,imbach@aeroscout.ch

web: www.aerosocut.ch, www.hslu.ch

KEY WORDS: UAVs, unmanned aerial vehicles, autonomous helicopter, aerial magnetic sensing, flight mission planning, sensor integration

\begin{abstract}
:
This paper concentrates on aerial magnetic sensing with an autonomous Scout B1-100 UAV helicopter. A high-resolution 3-axis magnetic sensor has been mounted on the helicopter in order to generate a detailed magnetic map and to identify various ferrous objects in the soil. The development is based on advanced mission planning for the UAV as well as test flights under challenging weather conditions such as wind gusts and snow fall. Finally, this paper summarizes a real-world application after the collapse of a daylight coal mining where various persons have been killed and multiple infrastructure objects have been buried. The task of magnetic scanning was applied in order to find buried vehicles where miners have been expected based on eyewitnesses during the collapse. However, while several ferrous objects have been located, the van could not be identified in the extensive area of the landslide.
\end{abstract}

\section{INTRODUCTION}

It is well-known that during the last decade UAV helicopters have achieved special recognition for various civil applications. Within a joint project between the Swiss company Aeroscout $\mathrm{GmbH}$ (www.aeroscout.ch) and the German company Mobile Geophysical Technologies (www.mgt-geo.com) it has successfully been demonstrated, that the autonomous unmanned Scout B1-100 UAV helicopter can be used for precise aerial magnetic sensing. A real life test for the integrated system was performed during multiple flight missions in Turkey during February 2011 after an open mining site has collapsed as shown in Figure 1 and several mine workers have been killed. This paper will shortly describe the technical challenges of the integration of the high-resolution magnetic system, the UAV mission planning, and the data analysis. A short section at the end summarizes the lessons learned.

The task of aerial magnetic scanning is based on two disciplines which historically have less connection. On the one hand there are the mechanical and electrical engineers as well as computer science specialists which have dominantly influenced and driven the UAV system integration (mechatronics), and on the other hand there are physicians and geophysicists which have strong experience in geophysical data acquisition and data processing, including magnetic data analysis or electro-magnetic signal distribution affected by the Earths' surface or different layers and objects of varying materials.

Therefore the first challenge for this project is to understand each other in terms of technical terms, technical requirements, and technical restrictions in general. Again, the question of 'realtime' has to be clarified and obviously there are different understandings of sensitivity, noise intensity and noise patterns, or ac-

${ }^{*}$ Dr. sc. techn. ETH in Electrical Engineering, General Manager of Aeroscout GmbH, Professor for Control Theory at the Lucerne University of Applied Sciences and Arts, AIAA Senior Member since 1996

${ }^{\dagger} \mathrm{BSc}$ in Electrical Engineering of Lucerne University of Applied Sciences and Arts, Group Leader at Aeroscout GmbH ceptable and non-acceptable disturbances. The open discussion and several flight tests have turned out that there are a few key challenges for a successful UAV system solution. Two of these challenges are described in more detail below, i.e., the sensor integration and the mission planning.

\section{SENSOR INTEGRATION}

The autonomously flying Scout B1-100 UAV helicopter is used as the sensor carrying platform. A picture of this helicopter is shown in Figure 2. This helicopter has a main rotor diameter of $3.20 \mathrm{~m}$, a payload weight of approx. $30 \mathrm{~kg}$ including $10 \mathrm{l}$ of fuel, the flight control system (FCS), the FCS batteries, and the radio modules, and a maximum take-off weight (MTOW) of approx. $75 \mathrm{~kg}$. The flight endurance under hover flight conditions at $600 \mathrm{~m}$ above mean sea level (AMSL) out of ground effect reaches up to $90 \mathrm{~min}$. Further details can be found on the website at www.aeroscout.ch. In its standard configuration, the Scout B1-100 UAV is equipped with the wePilot 1000 flight control system, which has originally been developed at the Swiss Federal Institute of Technology (ETH) Zurich described in References (Eck, 2001), (Chapuis et al., 2000)) and is being commercialized since several years by the company weControl AG, Switzerland with the companies' website www.wecontrol.ch. The implemented navigation algorithms are based on an extended Kalman filter as described previously in various papers such as Refernces (Eck and Geering, 2000), (Eck et al., 2000), (Ignagni, 1990), and (Ignagni, 1996).

While traditionally the magnetic sensor is carried by a person across the field of interest, it is now relevant that the sensor data acquisition can be performed remotely. During the flight of the UAV helicopter a direct access to the sensor and its working conditions is not possible. In addition, it had to be analyzed if and if yes, how the high-resolution magnetic sensor is affected by the environment of the UAV system, including effects from the engine, the onboard computers, the various data links, or other sen- 

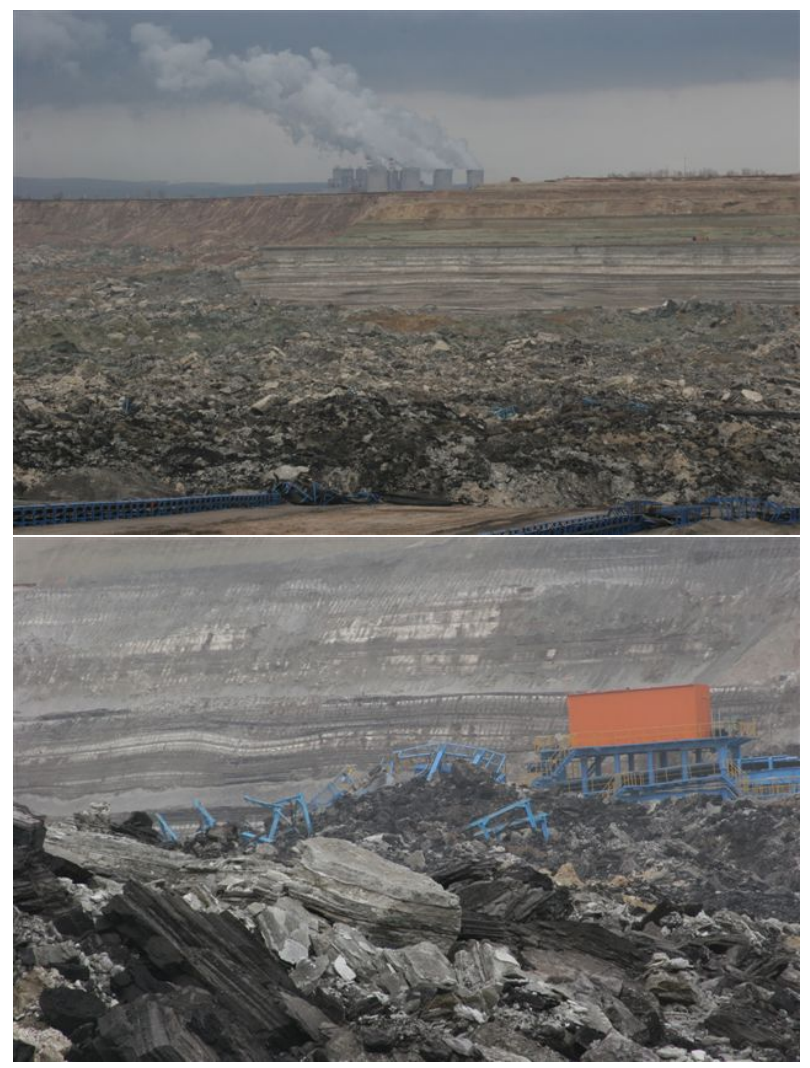

Figure 1: Overview of a real-world accident where a complete daylight coal mining site has collapsed. Several people have been killed and many objects have been buried.

sors and payload components. Beside the data access from the sensor, powering of the magnetic sensor is not trivial, as varying supply voltages can strongly affect the sensor readings. A special solution had to be found for the sensor in concern of vibration isolation due to the various sources of vibration patterns from the engine, the main rotor, and the tail rotor. The solution developed has placed the sensor in a certain distance from the helicopter main body, and providing a customized electronics for the sensor power supply and sensor data acquisition. A picture of the data acquisition module which records the magnetic data during the flight is shown in Figure 3. A simple button allows to start and stop the data acquisition. The LED indicates the status of the data logger. The logging configuration can be done by using a hyper terminal which is connected to the serial RS232 port. The overall weight of the logger module is approx. $200 \mathrm{gr}$. Further details on the embedded software architecture can be found in References (Styger, 2009) and (Styger, 2010).

\section{AUTOMATIC MISSION PLANNING}

In most commercial outdoor UAV applications the absolute flight precision of the UAV can vary between a few meters in altitude and horizontal accuracy. However, the flight accuracy for precise aerial magnetic scanning had to be improved below $0.5 \mathrm{~m}$. Due to this requirement a DGPS system was used in parallel with the inertial navigation sensor unit (IMU). Due to the fact that a very close line spacing for the field or region of interest was required, an algorithm has been developed which allows the UAV to make turns with respect to its physical limitation. This algorithm also takes into account that the payload (sensor) could also be mounted as a pendulum below the helicopter. The waypoints computed for the flight mission allow to cover the complete flight field and are providing a reference velocity during the magnetic

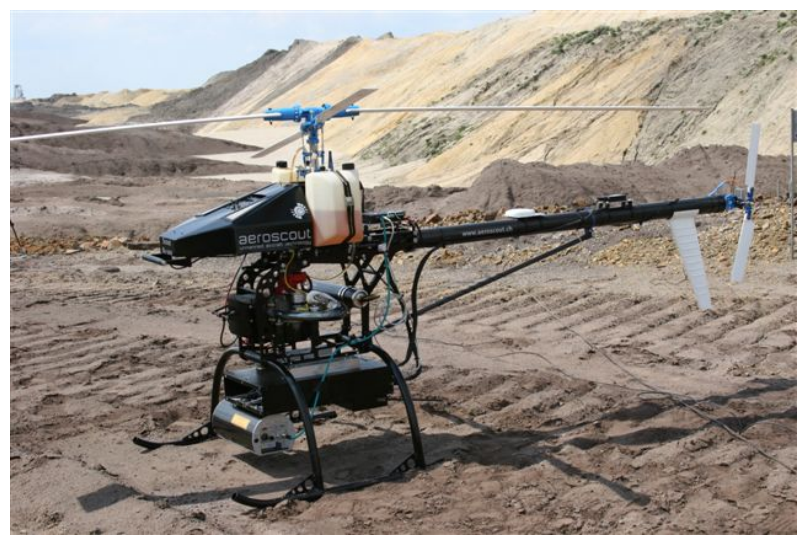

Figure 2: Picture of the Scout B1-100 UAV helicopter during airborne 3D laser flight testing in Germany. For these tests a RIEGL laser scanner LMS Q160, an onboard computer system, a broadband data link, and a DGPS system with a total payload weight of approx. $15 \mathrm{~kg}$ have been attached to the helicopter. The average flight endurance with $10 l$ of fuel is $1.5 \mathrm{~h}$.

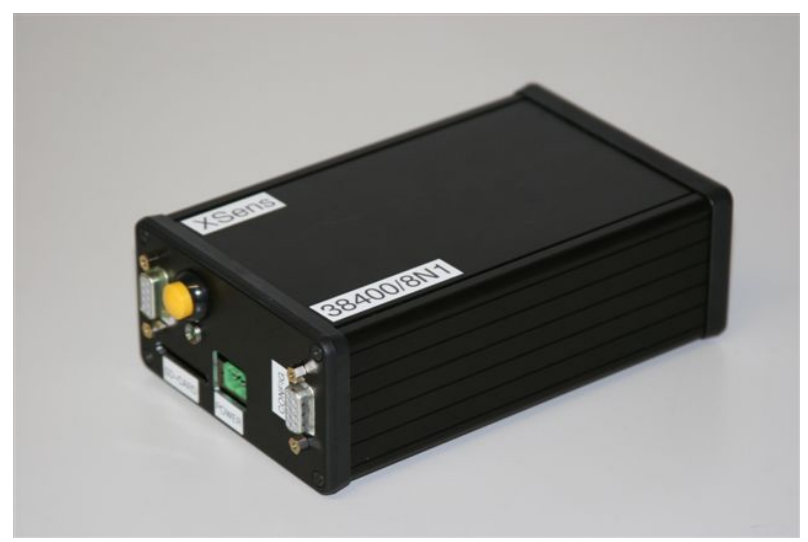

Figure 3: Picture of the magnetic data acquisition module developed at Lucerne University for Applied Sciences and Arts.

scan depending on the data acquisition rate of the magnetic sensor. Various test flights have been performed with a line-spacing of $1 \ldots 2 \mathrm{~m}$.

The application of precise autonomous aerial magnetic scanning defines various specific requirements. The goal of this application is to create a 3D magnetic map of the flight area of interest. In order to optimize the resolution of small ferrous objects in the soil it requires movement of the sensor in a $1 \ldots 2 \mathrm{~m}$ distance above the surface. Due to the extremely small clearance between sensor and ground this is an extraordinary task for an UAV since unmanned helicopters are used to fly at much higher altitudes. One of the reasons is of course the risk of collision with any object and another reason is the ground effect causing different aircraft behavior than at higher altitudes. In addition to this requirement some further challenges define the success of this UAV application:

- During the magnetic scanning process, the magnetic sensor should have almost none or very few pendulum movements.

- If there are movements of the magnetic sensor, these movements should be known.

- UAVs enable using drape flying in which the data is collected at constant terrain clearance.

- The magnetic sensor should not rotate around its own axis. 
- The exact position and orientation of the magnetic sensor must be known in order to plot the magnetic map.

- Line spacing between to two flight lines should be less than $2 m$ if possible.

- During the scanning process, the flight velocity shall be constant.

- Depending on the data rate of the magnetic scanner, the (horizontal) flight velocity shall be selectable.

Beside these requirements, some additional concerns have to be taken into account. One is to minimize the flight time of unnecessary paths, e.g., at the end of the flight field, when entering the next flight line.

Obviously, the curvature in the turn region, as well as the region of deceleration and acceleration of the UAV is optimized in order to reduce time while turning the UAV as well as to avoid strong motion of the sensor below of the helicopter. Furthermore, the UAV flight control system has to compensate for changing crosswind, headwind or following wind.

\subsection{Detailed Mission Planning}

In order to achieve the above mentioned requirements, a MATLAB ${ }^{T M}$ tool has been developed with the goal to automatically generate an appropriate mission profile.This process is described in more detail below. In this application, the flight area of interest was

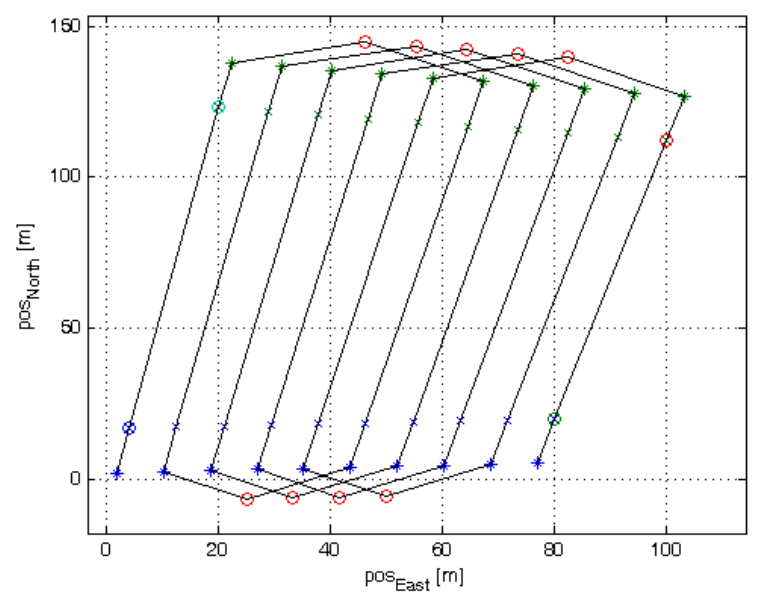

Figure 4: Initial mission planning with automatically defining GPS waypoints in order to receive a predefined distance of the flight lines above the complete flight area. The final GPS waypoint in this example can be seen in the picture in the lower right corner.

defined by four GPS coordinates given in the WGS-84 data format. The coordinates have been transformed to a local Cartesian coordinate system with north, east, down axes. As shown in Figure 4 , the flight area could then easily be filled with flight lines at a desired distance between each other, e.g., with the following arbitrarily chosen parameters

$$
\begin{aligned}
\delta B & =9 \mathrm{~m} \\
\delta S & =15 \mathrm{~m} \\
\delta R & =10 \mathrm{~m}
\end{aligned}
$$

with the distance between two flight lines $\delta B$, the distance to accelerate and to decelerate the helicopter $\delta S$, and the rough radius for the turn at the end of each flight line $\delta R$. Based on this approach each flight line contains four points, i.e., the first starting point to accelerate the helicopter, the second point when constant velocity has been achieved, the third point when deceleration of the helicopter begins, and the forth point when the velocity to start the turn has been achieved. Afterwards, the turn flight of the helicopter is based on one additional point before the next flight line begins. However, this simple approach becomes unpractical when the line spacing becomes only $\delta B=1 \ldots 2 \mathrm{~m}$. In this case, a substantial part of the overall flight time is lost during the turns.

\subsection{Advanced Mission Planning}

Again, the flight area is defined by four corner coordinates. The flight area is then divided into multiple segments with a width defined by $\delta A$. The influence of this new parameter is shown in Figure 5. In our case

$$
\delta A \approx 10 \ldots 15 m
$$

was selected and turned out as an appropriate width for the flight segments. In Figure 5 two flight segments are illustrated. The

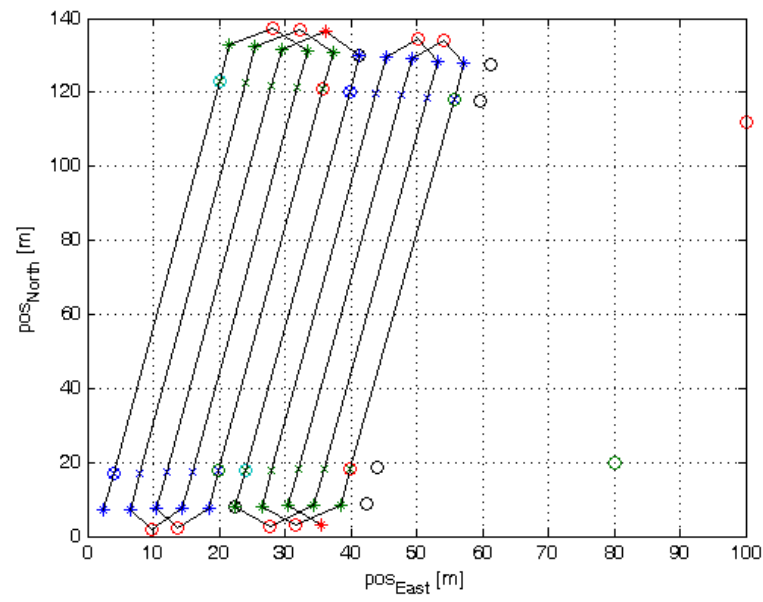

Figure 5: Advanced mission planning with additional segmentation of the flight area in order to avoid unnecessary flight time at the end of the area of interest.

plot shows that the amount of undesired flight time at the end of the flight area has been reduced considerably. This can also be seen in Figure 6 when the line spacing is further reduced by $\delta B=1 \mathrm{~m}$. Multiple field segments can easily be attached until the complete flight area has been covered. Further details on this approach can also be found in Reference (Eck, 2011).

From this example it can be seen that the number of generated waypoints has already reached

$$
n_{\text {waypoints }}=520
$$

for a small flight area when the field of interest is filled with a line-spacing of $1 \mathrm{~m}$. It would be very inefficient if these numerous waypoints would be handled successive or individually. The given parameters for this mission planning are given in equations (1) and (2) and can be adapted according to the magnetic scanning requirements and the UAV characteristics. Based on the computed waypoints for the desired mission profile, the waypoints must afterwards be translated into a mission structure which can then be uploaded to the flight control system. 


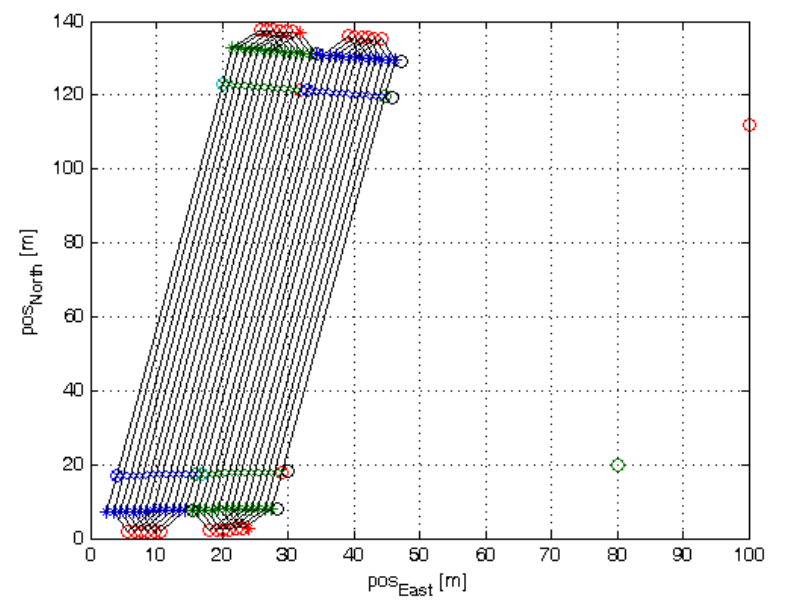

Figure 6: Advanced mission planning with additional segmentation of the flight area and very small line spacing.

\section{APPLIED FLIGHT MISSION PLANNING AND FLIGHT PERFORMANCE ANALYSIS}

This section describes and visualizes in some detail the recorded mission flight data ${ }^{1}$. The main results of the implementation of the semi-automated mission planning can be seen in Figure 7. The figure shows the 2D plot of the actually flown flight trajec-

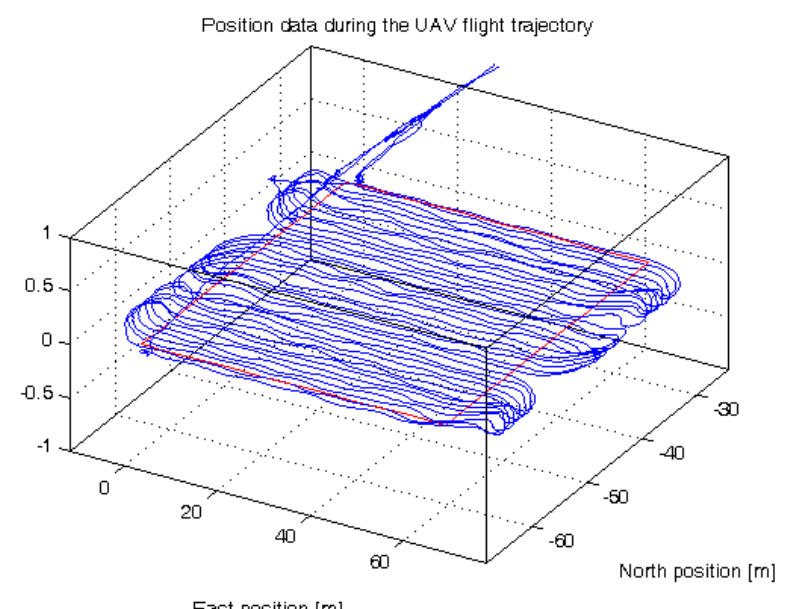

East position [m]

Figure 7: The actual recorded UAV position data during the aerial magnetic scanning mission.

tory (blue line) and the desired scanning area (red line). The flight profile shows that the turns of the UAV system occur at the end of the scanning area. While the UAV is flying within the scanning area a constant velocity and orientation is maintained. The flight velocity data is visualized in Figure 8. As wind is coming from one direction, the turns of the UAV clockwise and counterclockwise look slightly different. Figure 7 also shows that the scanning area during this 40 minutes UAV flight is well covered. In Figure 8 the UAV attitude data is presented. While the yaw (heading) angle only changes during the turns, the pitch angle is affected by the influence of the wind. Obviously, one direction

\footnotetext{
${ }^{1}$ The autonomous aerial magnetic scanning test flights with the Scout B1-100 UAV helicopter were performed during December 2010 in a test area in Germany. In this flight area various objects of different sizes and types were located below the surface and were not visible for human eyes. The processing of the magnetic scanning data has been published by Dr. J. Stoll, MGT, Germany.
}
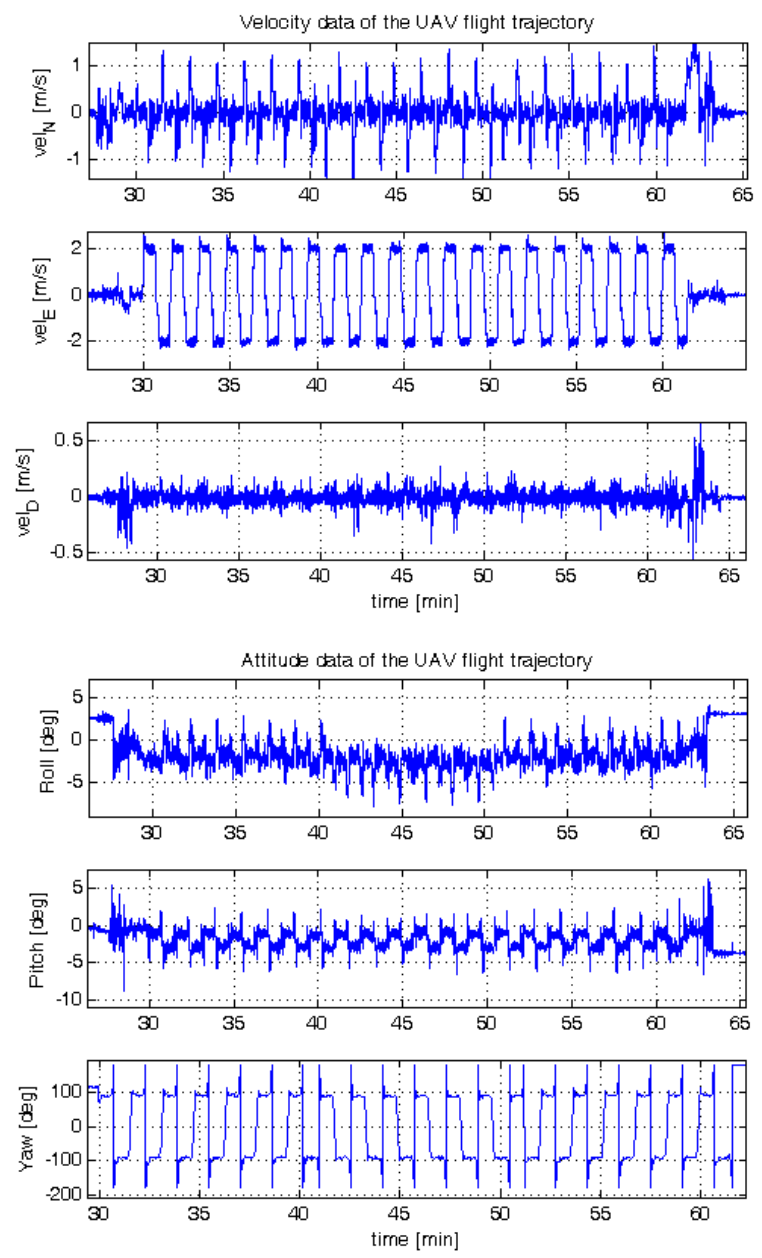

Figure 8: The actual recorded UAV velocity and attitude data during the aerial magnetic scanning mission.

has been flown with and one direction against the wind direction. The roll angle only changes slightly during the turns of the UAV helicopter. These changes are also influenced by the wind and partly influenced by the small oscillations of the magnetic payload pendulum.

With respect to the mentioned requirements as given in Section 3 it turns out that the mission planning and the data received has been successful. There were almost no pendulum movements of the magnetic sensor which allows to identify ferrous objects clearly. This could be seen in the magnetic data which show magnetic deviations at the same location when passing or approaching the object several times, e.g. when the object is located between two flight lines. Analyzing the flight altitude shows, that the altitude variation is within centimeter accuracy. Looking on the magnetic data, this allows to draw conclusions on the size of the ferrous object below the surface. A more detailed analysis of the magnetic data also shows that there is a homogeneous distribution of magnetic points covering the flight area. This was only achievable with a constant scanning velocity as shown in Figure 8.

\section{REAL-WORLD FIELD EXPERIENCE}

Initial flight tests for aerial magnetic scanning with Aeroscouts' Scout B1-100 UAV helicopter have been performed in Switzerland and Germany. It was possible to create a magnetic map of a region of interest based on the flight profile of the UAV. After a 
landslide at an open-mining in Turkey in February 2011, various cars and vans have been buried. Pictures of the site can be seen in Figure 1.

After the detailed investigations at the location, the region of in terest could be defined. During multiple flight missions, a magnetic map of the region of interest could be created with the Scout B1-100 UAV helicopter equipped with the magnetic sensor. Pictures of the helicopter during the automatic aerial magnetic sensing process can be seen in Figure 9. After the mis-

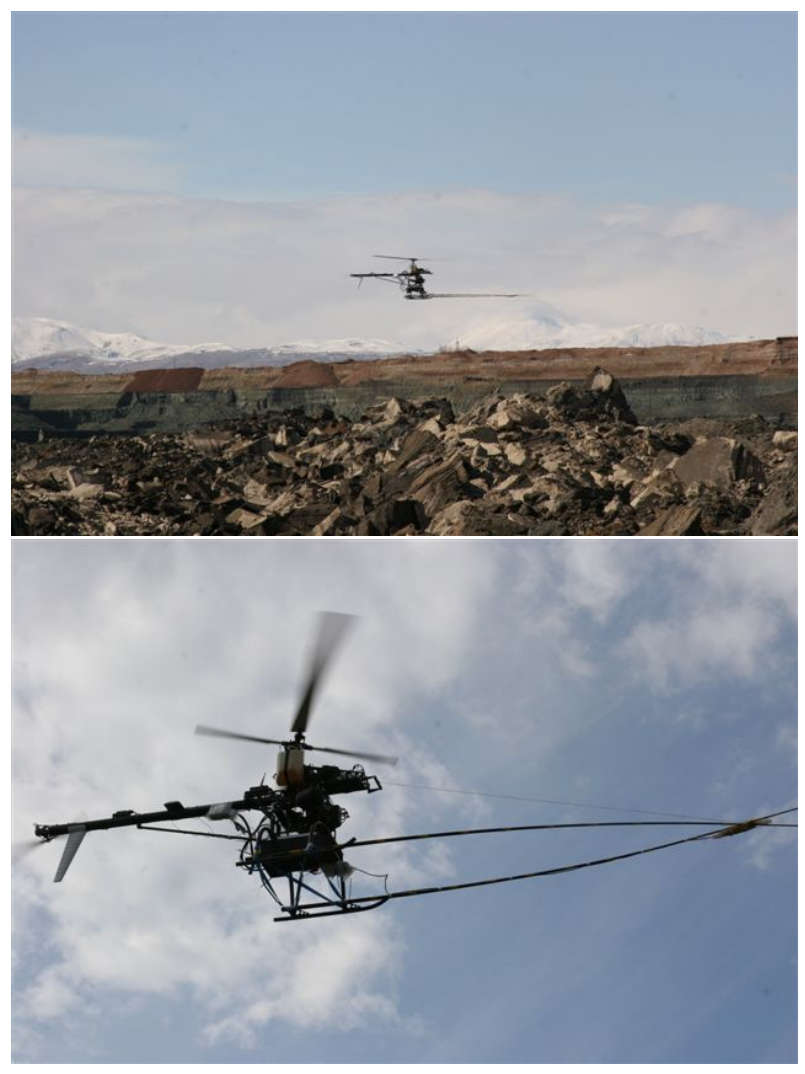

Figure 9: Applied automatic mission flight for aerial magnetic sensing at the coal mining site mentioned above.

sion flights, the magnetic map of the area could be generated (www.mgt-geo.com). The map has shown various locations of magnetic objects. As a remaining technical challenge was to further analyze the magnetic data in order to decide which kind of object at which distance below the surface could be identified and dredged.

\section{SUMMARY AND LESSONS LEARNED}

The project described above was jointly fulfilled between Aeroscout, Switzerland and MGT, Germany. Fruitful discussions between engineers and physicians have yielded a successful UAV system integration which fulfills the flight performance and realtime data acquisition in order to generate a precise magnetic map of a region of interest. The aerial magnetic data acquisition is based on a complete autonomous flight profile as required by the integrated magnetic sensor. During the development process it has turned out that the ability of multiple flight tests with different system configuration was necessary. Also, reference data from other solutions such as traditional walking across the field with the magnetic sensor was helpful. For the UAV mission development it turned out that the real-time data recording and the ability for data post-processing were relevant tools.

\section{CONCLUSIONS}

This paper gives a detailed overview on a new UAV application, namely the mission planning for autonomous aerial magnetic sensing. UAV test flights under real geophysical surveying conditions with the Scout B1-100 UAV helicopter were accomplished based on the mission profiles described herein. The successful mission implementation and realization are shown with the actual recorded flight data. This flight data shows a high accuracy for the altitude performance and a dense coverage of the complete flight area with the magnetic sensor. Although the test flights were performed under challenging weather conditions (heavy snowfall, minus $7^{\circ} \mathrm{C}$, gust of wind) and a pendulum payload mounted below the helicopter, the method has proven reliability for long UAV flights. The recorded mission data of a 45 min test flight has been presented.

\section{ACKNOWLEDGEMENTS}

This work was supported by the Lucerne University of Applied Sciences and Arts at the Competence Center (CC) Electronics led by Prof. Zeno Stössel. Only the partnership between both companies Aeroscout GmbH and Mobile Geophysical Technologies could enable this project and both companies have strongly supported the UAV field testing. We would like to thank Prof. Dr. Roland Siegwart, Vice President of the Swiss Federal Institute of Technologies (ETH) Zurich, Switzerland, and his research group for their continuous support of our UAV activities. Finally, this paper is dedicated to the mine workers who have lost their life during the landslide. We express our condolence to the involved families. May this tragedy further support the usage and the development of civil UAVs.

\section{REFERENCES}

Chapuis, J., Eck, C., Kottmann, M., Sanvido, M. and Tanner, O., 2000. Control of Complex Systems. Springer.

Eck, C., 2001. Navigation Algorithms with Applications to Unmanned Helicopters. PhD thesis, Swiss Federal Institute of Technology (ETH) Zurich, Diss. ETH No. 14402.

Eck, C., 2011. Mission planning and flight precision analysis for autonomous aerial magnetic scanning. AIAA Guidance, Navigation, and Control Conference.

Eck, C. and Geering, H. P., 2000. Error dynamics of model based INS/GPS navigation for an autonomously flying helicopter. Proceedings of the AIAA Guidance, Navigation, and Control Conference, Denver, CO, AIAA-2000-4465, pp. 1-9.

Eck, C., Chapuis, J. and Geering, H. P., 2000. Entwicklung einer Helikopterdrohne für den wissenschaftlichen Einsatz in 16. Fachgespräch "Autonome Mobile Systeme (AMS 2000), Karlsruhe. Dillmann, R. and H. Wörn and M. von Ehr (Hrsg), Springer.

Ignagni, M. B., 1990. Optimal strapdown attitude integration algorithms. Guidance, Control, and Dynamics 13(2), pp. 363-369.

Ignagni, M. B., 1996. Efficient class of optimized coning compensation algorithms. Guidance, Control, and Dynamics 19(2), pp. 424-429.

Styger, E., 2009. Ecsd - reusable embedded software components. Embedded Computing Conference 2009.

Styger, E., 2010. Freertos as reusable software component. Embedded Computing Conference 2010. 